\title{
Impedance Characteristics of a Uniform Current Loop Having a Spherical Core
}

\author{
Saburo Adachi ${ }^{1}$
}

(November 12, 1959)

\begin{abstract}
The radiation impedance is derived by the electromotive force method in a convenient form as the sum of the self-radiation impedance of a loop in the free space and an additional term due to the reaction between the loop and the sphere which is proportional to the wellknown expansion coefficient of a magnetic-type scattered wave from a sphere in an incident plane wave. The first antiresonance frequency has been given in the form of a universal curve for a very small uniform current loop with core of an arbitrary composition of $\mu_{s}$ and $\epsilon_{s}$, subject to the condition that the refraction coefficient $N=\sqrt{\mu_{s} \epsilon_{s}}$ is extremely large. Some numerical calculations show that high $-\mu$ core is desirable for a comparatively lower frequency region, and high $-\epsilon$ core is rather desirable in an antiresonance region.
\end{abstract}

\section{Introduction}

By use of a sufficiently high refractive index, large scattering or absorption cross sections may be obtained from spheres small in terms of wavelength. For example, it has been shown that the echoing area can be increased to approximately three-quarters of a square wavelength for resonance of the electric or magnetic dipole type, and the required index of refraction can be given as a function of sphere size.

In a small loop antenna the ohmic resistance normally exceeds the radiation resistance. An increase in radiation resistance is highly desirable to improve the radiation efficiency. It can be expected that a high induced voltage, and therefore a highradiation resistance, can be obtained by encircling a small resonant sphere by such a loop. Of course, it is desired that the radiation reactance be as low as possible. The ratio of the radiation resistance to the radiation reactance is also an important criterion.

Very recently, the most general theoretical analysis for a thin loop with a spherical core has been given by Herman [1] $]^{2}$ and Cruzan [2]. In these analyses, however, it is not easy to derive general relationships between the input impedance and the medium of the spherical core because of the difficulties of the numerical computations.

The purpose of the present paper is to correlate the medium constants of the core with the impedance characteristics under the assumption of a uniform current distribution. $^{3}$ In order to derive the radiation impedance, a conventional emf method is applied to the electromagnetic field solution of a uniform loop current in the presence of a sphere which has been

1 Antenna Laboratory, Department of Electrical Engineering, The Ohio State University, Columbus 10; Ohio; while author was on leave of absence from Tohoku University, Sendai, Japan (invited paper).

2 Figures in brackets indicate the literature references at the end of this paper.

3 The assumption of constancy of current in the loop requires that the loop 3 The assumption of constancy of current in the loop requires that the loop
be fed at a number of points around the loop. In view of the large magnitude of $N k_{o} a$ a localized generator would produce a highly nonuniform current. treated independently by Tai [3] and Wait [4]. The radiation impedance is given as the sum of the self-radiation impedance of the air loop and an additional term due to the reaction between the loop and the sphere which is proportional to the wellknown expansion coefficient of the magnetic-type wave scattered from a sphere in an incident plane wave.

\section{Impedance Characteristics of a Uniform Current Loop Having a Spherical Core}

The general solution for the electromagnetic field due to a uniform current loop in the presence of a sphere has been given by C. T. Tai. Geometrical configurations are illustrated in figure 1 . The radii of the sphere and the loop are the same and are

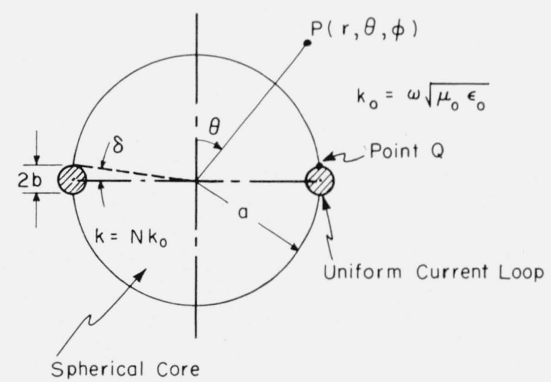

Figure 1. Uniform current loop having a spherical core.

indicated by $a$; therefore, the wire of the loop is partially immersed into the core. Wavenumbers of the free space and the medium of the sphere are indicated by $k_{0}$ and by $k=N k_{0}\left(N=\sqrt{\epsilon_{s} \mu_{s}}\right)$, respectively. As the time dependence, $e^{j \omega t}$ is used. The primary fields due to the uniform loop current, $I$, are expressed by the following equations: 


$$
\begin{aligned}
& \bar{E}^{i}=\sum_{n=1}^{\infty} C_{n} \bar{M}_{\text {eon }}^{(1)}\left(k_{0}\right) \\
& \bar{H}^{i}=\frac{k_{0}}{j \omega \mu_{0}} \sum_{n=1}^{\infty} C_{n} \bar{N}_{\text {eon }}^{(1)}\left(k_{0}\right)
\end{aligned}
$$

and

$$
\begin{aligned}
& \bar{E}^{i}=\sum_{n=1}^{\infty} C_{n} \frac{j_{n}(\alpha)}{h_{n}^{(2)}(\alpha)} \bar{M}_{\text {eon }}^{(4)}\left(k_{0}\right) \\
& \bar{H}^{i}=\frac{k_{0}}{j \omega \mu_{0}} \sum_{n=1}^{\infty} C_{n} \frac{j_{n}(\alpha)}{h_{n}^{(2)}(\alpha)} \bar{N}_{\text {eon }}^{(4)}\left(k_{0}\right)
\end{aligned}
$$

where $\alpha=k_{0} a$, and

$$
C_{n}=-\frac{I \omega \mu_{\gamma} \alpha}{2} \frac{2 n+1}{n(n+1)} P_{n}^{1}(0) h_{n}^{(2)}(\alpha) .
$$

The vector wave functions $\bar{M}_{\text {eon }}$ and $\bar{N}_{\text {eon }}$ are the same with $\bar{m}_{\text {eon }}$ and $\bar{n}_{\text {eon }}$ defined in the text by Stratton [5]. The fields reflected to the external region and the field transmitted into the sphere are, respectively, given by

$$
\left.\begin{array}{ll}
\bar{E}^{r}=\sum_{n=1}^{\infty} C_{n} R_{n} \bar{M}_{\text {eon }}^{(4)}\left(k_{0}\right) & \\
\bar{H}^{r}=\frac{k_{0}}{j \omega \mu_{0}} \sum_{n=1}^{\infty} C_{n} R_{n} \bar{N}_{\text {eon }}^{(4)}\left(k_{0}\right) &
\end{array}\right\}
$$

and

$$
\left.\begin{array}{ll}
\bar{E}^{t}=\sum_{n=1}^{\infty} C_{n} T_{n} \bar{M}_{\text {eon }}^{(1)}\left(N k_{0}\right) & \\
\bar{H}^{t}=\frac{k}{j \omega \mu} \sum_{n=1}^{\infty} C_{n} T_{n} \bar{N}_{\text {eon }}^{(1)}\left(N k_{0}\right) &
\end{array}\right\}
$$

the electric field at the point $Q$ (on the surface of the sphere, and at a distance $b$, the radius of the wire, from the current filament which is located at the equator of the sphere) as the average value of the electric field around the wire cross section; namely,

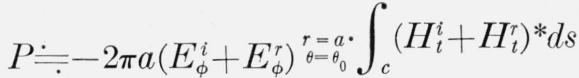

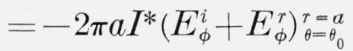

where

$$
\theta_{0}=\pi / 2-\delta \simeq \pi / 2-\frac{b}{a} .
$$

Thus, the radiation impedance obtained from the emf method is expressed by

$$
Z=\frac{P}{|I|^{2}}=Z_{0}+Z_{s}
$$

where

$$
\begin{aligned}
& Z_{0}=\pi \eta \alpha^{2} \sum_{n=1}^{\infty} \frac{2 n+1}{n(n+1)} P_{n}^{1}(0) P_{n}^{1}\left(\cos \theta_{0}\right) j_{n}(\alpha) h_{n}^{(2)}(\alpha) . \\
& Z_{s}=\pi \eta \alpha^{2} \sum_{n=1}^{\infty} \frac{2 n+1}{n(n+1)} P_{n}^{1}(0) P_{n}^{1}\left(\cos \theta_{0}\right) R_{n} h_{n}^{(2)}(\alpha)^{2} .
\end{aligned}
$$

In the above equations, $Z_{0}$ represents the radiation impedance of a loop without a spherical core, $Z_{s}$ represents the variation of the radiation impedance due to the reaction between the spherical core and the loop, and $\eta$ is the intrinsic impedance of free space. The imaginary part of series (10) does not converge rapidly enough when $\theta_{0}$ approaches $\pi / 2$, or the thickness of the wire decreases to zero. By using the asymptotic expression of Legendre and Bessel functions, the radiation reactance $X_{0}$ in (10) can be transformed into the following alternative equations :

$R_{n}=\frac{\mu_{0} j_{n}(\alpha)\left[N \alpha j_{n}(N \alpha)\right]^{\prime}-\mu j_{n}(N \alpha)\left[\alpha j_{n}(\alpha)\right]^{\prime}}{\mu j_{n}(N \alpha)\left[\alpha h_{n}^{(2)}(\alpha)\right]^{\prime}-\mu_{0} h_{n}^{(2)}(\alpha)\left[N \alpha j_{n}(N \alpha)\right]^{\prime}}$

$T_{n}=\mu \frac{j_{n}(\alpha)\left[\alpha h_{n}^{(2)}(\alpha)\right]^{\prime}-h_{n}^{(2)}(\alpha)\left[\alpha j_{n}(\alpha)\right]^{\prime}}{\mu j_{n}(N \alpha)\left[\alpha h_{n}^{(2)}(\alpha)\right]^{\prime}-\mu_{0} h_{n}^{(2)}(\alpha)\left[N \alpha j_{n}(N \alpha)\right]^{\prime}}$.

It is very interesting to note that the coefficient $R_{n}$ is exactly the same as the coefficient associated with the magnetic-type wave scattered by a sphere in an incident plane wave. The coefficients $R_{n}$ and $T_{n}$ express the effect of the spherical core on the performance of the loop antenna. Neither the electric field nor the magnetic field (the surface current) are uniform around the wire cross section. If, however, the wire is sufficiently thin, it is reasonable to assume

$$
\begin{aligned}
X_{0}=\eta \alpha & \left\{\ln \frac{a}{2 b}+\frac{\pi}{4} \frac{b}{a}\right\} \\
-\pi \eta \alpha^{2} & \sum_{n=1,3}^{\infty} \frac{1}{n+1}\left[\frac{2 n+1}{n} P_{n}^{1}(0) P_{n}^{1}\left(\cos \theta_{0}\right) j_{n}(\alpha) n_{n}(\alpha)\right. \\
& \left.+\frac{1}{\pi \alpha}\left\{2 \cos \frac{b}{a}(n+1)+\frac{b}{a} \sin \frac{b}{a}(n+1)\right\}\right] .
\end{aligned}
$$

The detailed derivation of the above equation is given in reference [6]. For a small loop, i.e., $\alpha \ll 1$, the radiation impedance is approximated by retaining the term $n=1$ only; namely, 


$$
Z_{0} \simeq 20 \pi^{2} \alpha^{4}+j 60 \pi \alpha\left\{2 \ln \frac{8 a}{b}-4.40\right\} .
$$

As for the constant $R_{n}$, many discussions have been made relating to the problem of the scattering of a plane wave by a sphere. These results can be directly applied to the present problem. For the lossy spherical core, i.e., the complex values of $N$, it is convenient for the computation to transform eq (11) by using the logarithmic derivative functions [7] with respect to spherical Bessel functions into the following form:

$$
\begin{array}{r}
Z_{s}=-\pi \eta \alpha^{2} \sum_{n=1}^{\infty} \frac{2 n+1}{n(n+1)} P_{n}^{1}(0) P_{n}^{1}\left(\cos \theta_{0}\right) j_{n}(\alpha) h_{n}^{(2)}(\alpha) \\
\times\left\{\frac{\sigma_{n}(\alpha)-N \frac{\mu_{0}}{\mu} \sigma_{n}(N \alpha)}{\rho_{n}(\alpha)-N \frac{\mu_{0}}{\mu} \sigma_{n}(N \alpha)}\right\}
\end{array}
$$

where $\rho_{n}(\alpha)$ and $\sigma_{n}(\alpha)$ are the logarithmic derivative functions with respect to spherical Hankel and Bessel functions, respectively, i.e.,

$$
\begin{aligned}
& \rho_{n}(x)=\frac{d}{d x} \ln \left[x h_{n}^{(2)}(x)\right] \\
& \sigma_{n}(x)=\frac{d}{d x} \ln \left[x j_{n}(x)\right] .
\end{aligned}
$$

The function $\sigma_{n}(N \alpha)$ of any order with complex arguments $N \alpha$ can be computed by the recurrence formula of the logarithmic derivative functions.

Consider the maximum value of $R_{n}$ and its condition. A discussion has been given for special cases of pure dielectric $\left(\mu_{s}=1\right)$ or magnetic $\left(\epsilon_{s}=1\right)$ materials by E. M. Kennaugh. General cases for an arbitrary composition of $\boldsymbol{\epsilon}_{s}$ and $\mu_{s}$ can be discussed in a similar way. When a sphere is sufficiently small in terms of wavelength, $\alpha \ll 1$, only the term $R_{1}$ is significant. It is concluded that the maximum absolute value of $R_{1}$ is -1 at the condition of

$$
\frac{\mu}{\mu_{0}} j_{1}(N \alpha)\left[\alpha n_{1}(\alpha)\right]^{\prime}-n_{1}(\alpha)\left[N \alpha j_{1}(N \alpha)\right]^{\prime}=0 .
$$

For $\alpha \ll 1$, the above equation is approximately rewritten in the following transcendental equation:

$$
\frac{1}{N \alpha}+\frac{N \alpha}{\mu_{s}-1}=\cot N \alpha
$$

This equation is graphically solved with respect to $N \alpha$. Figure 2 shows $N \alpha / \pi$ as a function of $\mu_{s}$. Since $\alpha$ is assumed to be very small compared to unity, $N$ is necessarily very large, i.e., $\sqrt{\mu_{s}} \epsilon_{s} \gg 1$. This value gives the first antiresonance frequency of a small uniform-current loop with a lossless spherical core having arbitrary values $\epsilon_{s}$ and $\mu_{s}$. It should be noted here that the above discussion can be applied to the problem of the plane wave scattering

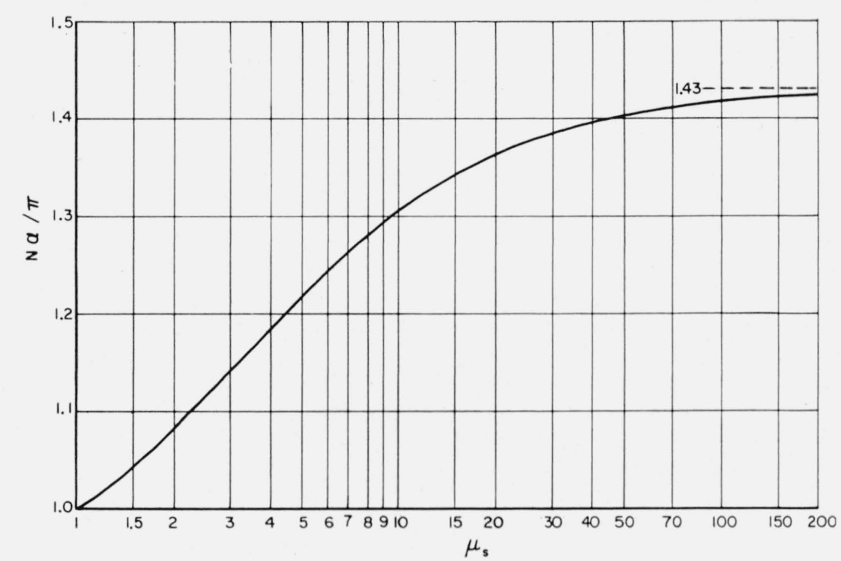

Figure 2. Antiresonance frequency of a uniform current loop having a spherical core with arbitrary constants $\mu_{\mathrm{s}}$ and $\epsilon_{\mathrm{B}}$, where $\mathrm{N} \alpha=\sqrt{\mu_{\mathrm{s}} \epsilon_{\mathrm{s}}} \mathrm{k}_{0} \mathrm{a}=\mathrm{ka}$.

by a small sphere; namely, the curve of figure 2 gives the magnetic-type resonance frequency of the sphere, and also gives the electric-type resonance frequency by replacing $\mu_{s}$ by $\epsilon_{s}$.

The maximum radiation resistance at the antiresonance point is given by

$$
R=R_{0}+R_{s}=\frac{3}{2} \pi \eta \alpha^{2} n_{1}(\alpha)^{2} \simeq \frac{3}{2} \pi \eta \frac{1}{\alpha^{2}},(\alpha \ll 1) .
$$

The maximum resistance increases in the order of $\alpha^{-2}$ with the decrease of $\alpha$. It should be noted that in an actual one-point-fed loop, unless infinitesimally small, the frequency of its antiresonance deviates from that predicted by figure 2 due to the nonuniform current distribution. This situation is illustrated in table 1.

The diameter of the antenna in terms of a wavelength in the medium at an antiresonance is found to vary between 0.159 and 1.43 by the constants of a medium. The results in figure 2 give the limiting case of an infinitesimally small loop, i.e., $\mu_{s} \epsilon_{s} \rightarrow \infty$. Similarly, the expression for $Z$ in eq (9) is correct only for an infinitesimally small loop, if it is fed at one point. The radiation impedance $Z$ has been calculated for the two cases $\epsilon_{s}=100, \mu_{s}=1$, and $\mu_{s}=100$,

\begin{tabular}{|c|c|c|}
\hline Medium of core & $\alpha$ & $2 a$ \\
\hline $\begin{array}{l}\text { Air } \\
{ }^{*} \mu_{s}=10.8-j 0.24 \\
\epsilon_{8}=9.5-j 0.077 \\
\left(\mu_{8} \epsilon_{8}\right) \rightarrow \infty\end{array}$ & $\left\{\begin{array}{l}0.5 \\
\frac{\pi}{\sqrt{\mu_{s} \epsilon_{s}}}(1.00 \sim 1.43)\end{array}\right.$ & $\begin{array}{l}0.159 \lambda \\
.496 \lambda \\
(1.00 \sim 1.43) \lambda\end{array}$ \\
\hline
\end{tabular}
$\epsilon_{s}=1$. For simplicity, the core is assumed to be lossless. Figure 3 and 4 show the radiation resistances and the radiation reactances. It must be noted here also, as mentioned before, that these figures do not give quantitative results for a one-point-fed loop be-

TABLE 1. Relation between medium of core and dimensions at antiresonance

*From ref [1] by Julius Herman, $\lambda$ is the wavelength in the medium of the core. 


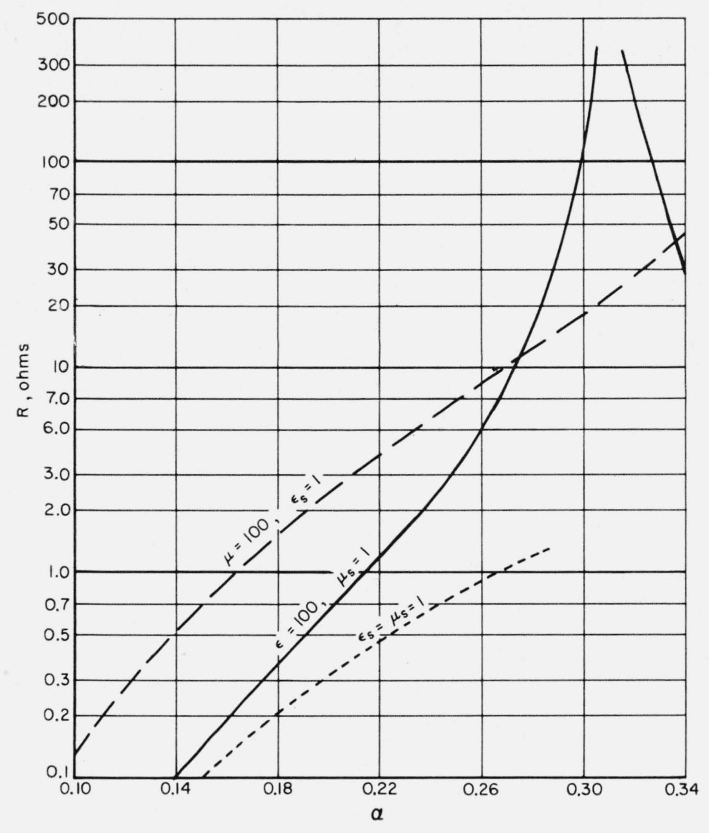

Figure 3. Radiation resistances of uniform current loops having air, dielectric, and magnetic cores $(\mathrm{N}=1,10 ; \mathrm{a} / \mathrm{b}=60)$.

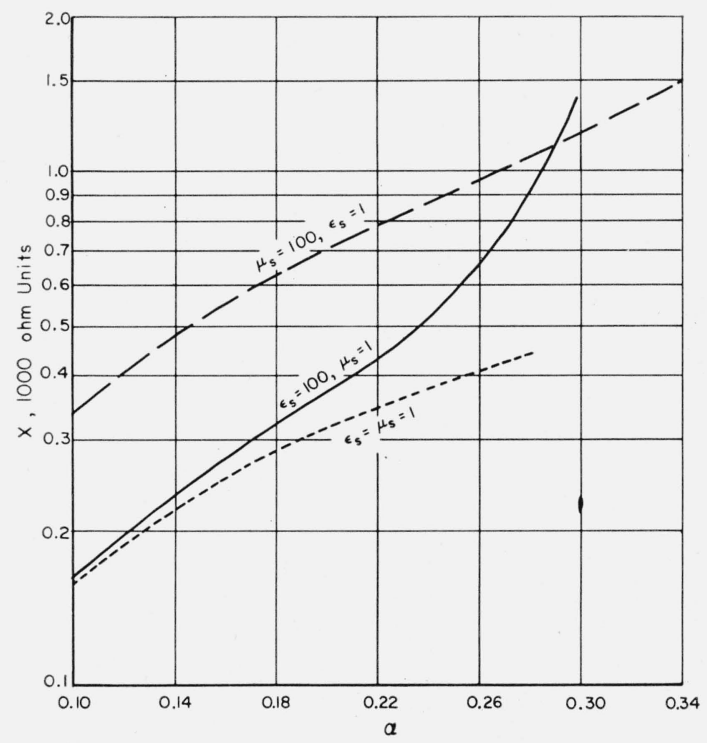

FIgURE 4. Radiation reactances of uniform current loops having air, dielectric, and magnetic cores $(\mathrm{N}=1,10 ; \mathrm{a} / \mathrm{b}=60$.)

cause of its nonuniform current. As for the case of $\epsilon_{s}=100$, the antiresonance occurs at $\alpha=\pi / 10$, and the curves approach asymptotically to those of an air loop with the decrease of $\rho$. The $\mu$-core is effective in order to increase the radiation resistance in the lower frequency region. The radiation impedance for an arbitrary composition of $\mu$ and $\epsilon$ ought to show a behavior intermediate between these two extreme cases. In figure 5 the ratios of the radiation resistances to the radiation reactances are plotted. From these figures it can be concluded that high- $\mu$ core is

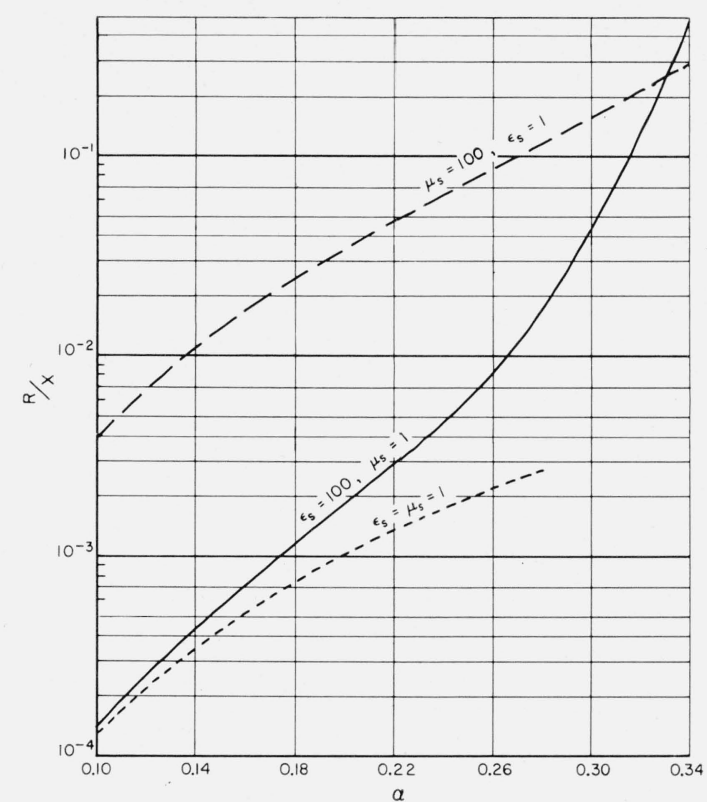

Figure 5. Ratios of radiation resistances to radiation reactances of uniform current loops having air, dielectric, and magnetic cores $(\mathrm{N}=1,10 ; \mathrm{a} / \mathrm{b}=60)$.

desirable for a comparatively low-frequency region, and high- $\epsilon$ core is rather desirable in order to use it in a antiresonant frequency region. This can be said also from the viewpoint of medium losses, that is, losses of usual magnetic materials increase more rapidly with the frequency than do the losses of dielectric materials.

\section{Conclusion}

The impedance characteristics of a uniform current loop (see footnote 3 ) with a spherical core of an arbitrary composition of $\mu$ and $\epsilon$ have been theoretically investigated. The radiation impedance is derived by the emf method in a convenient form as the sum of the self-radiation impedance of an air loop and an additional term due to the reaction between the loop and the sphere which is proportional to the well-known expansion coefficient of a magnetic-type scattered wave from a sphere in an incident plane wave. The first antiresonance frequency has been given in the form of a universal curve for a very small uniform current loop with a core of an arbitrary composition of $\mu_{s}$ and $\boldsymbol{\epsilon}_{s}$, subject to the condition that the refraction coefficient $N=\sqrt{\mu_{s} \epsilon_{s}}$ is extremely large. It should be noted that the above results can be directly applied to the first magnetic-type resonance frequency of a sphere in an incident plane wave. To show quantitative impedance characteristics of a loop with an arbitrary composite core, the radiation impedances for two cases, namely $\epsilon_{s}=100, \mu_{s}=1$, and $\mu_{s}=100, \boldsymbol{\epsilon}_{s}=1$, have been calculated and compared with an air loop. It is concluded that high- $\mu$ core is desirable for a comparatively lower frequency region, and high- $\epsilon$ core is rather desirable in an antiresonance frequency region. 
The author thanks E. M. Kennaugh for suggesting this investigation as well as for guidance in the course of the work, and also Dr. R. G. Kouyoumjian for his valuable discussions.

\section{References}

[1] J. Herman, Thin wire loop and thin biconical antennas in finite spherical media, Ph. D. Dissertation, University of Maryland, Publication No. 25, 331 (1957); Further extensions of loop antenna theory, TR-756 (July 1957), Diamond Ordnance Fuze Laboratory.

[2] O. R. Cruzan, Radiation properties of a spherical ferrite antenna, TR-387 (Oct. 1956), VHF ferrite antenna radiation properties, TR-516 (Aug. 1957), Diamond Ordnance Fuze Laboratory.

[3] C. T. Tai, Radiation from a uniform circular loop antenna in the presence of a sphere, Stanford Research Instit. Tech. Rept. No. 32 (1952).
[4] J. R. Wait, Electromagnetic coupling between a circular loop and a conducting sphere, Geophysics 18, 971 (1953); see also Ph. D. Thesis, University of Toronto (March 1951). The loop inside a spherical cavity in a dissipative medium has also been considered by J. R. Wait, Insulated loop antenna immersed in a conducting medium, J. Research NBS 59, 133 (1957) RP2781.

[5] J. A. Stratton, Electromagnetic Theory (McGraw-Hill Book Co., Inc., New York, N.Y., 1941).

[6] S. Adachi, Impedance characteristics of a uniform current loop having a spherical core, The Ohio State University Antenna Lab. Tech. Rept., 662 (1959).

[7] A. L. Aden, Electromagnetic scattering from sphere with sizes comparable to the wavelength, J. Appl. Phys. 22, 601 (1951).

Boulder, Colo.

(Paper 64D3-61)

\section{Publication of the National Bureau of Standards*}

\section{Selected Abstracts}

\section{The effect of multipath distortion on the choice of operating frequencies for high-frequency communi- cation circuits, D. K. Bailey, IRE Trans. Ant. Prop.} $\mathbf{A P}-\mathbf{y}, 397$ (1959).

Harmful multipath distortion on high-frequency facsimile services and telegraphic services operating at high speeds occurs when the received signal is composed of two or more components arriving by different modes over the same greatcircle path with comparable intensities, but having travel times which differ by an amount equal to an appreciable fraction of the duration of a signal element. The dependence of multipath distortion on the relationship of the operating frequency to the MUF is discussed and a new term, the multipath reduction factor (MRF), is introduced which permits calculation in terms of the MUF of the lowest frequency which can be used to provide a specified measure of protection against multipath distortion. The MRF has a marked pathlength dependence and is calculated as a function of path length for representative values of the other parameters involved by making use of an ionospheric model. It is then shown how the MRF can be used in connection with worldwide MUF prediction material to determine the minimum number of frequencies which must be assigned to a high-frequency communication service of continuous availability operating at high speed. Some comparisons with observations are discussed, and finally conclusions are drawn concerning manner of operation and choice of operating frequencies to reduce or to eliminate harmful multipath distortion.

\section{Reception of space diversity transmitters-Observa- tions over long-distance path to evaluate the useful- ness of the system, J. W. Koch, Wireless World 65, $512(1959)$.}

The British Broadcasting Corporation recently provided a series of transmissions to test the effectiveness of transmitter space-diversity on reception at distant points. Both very widely-spaced and relatively closely-spaced transmitters were used in the tests. The transmissions were at a frequency of $9,510 \mathrm{kc} / \mathrm{s}$, directed towards the east coast of the United States. Transmitting conditions were switched at intervals of approximately fifteen minutes during the testing period each day.

Observations of the received signals were made at the National Bureau of Standards Laboratories in Boulder, Colorado, from November 3, 1958 to November 14, 1958. The recordings obtained during these observations have been analyzed for fading characteristics and intelligibility, and the results of the analysis are given in this article.
Mismatch errors in cascade-connected variable attenuators, G. E. Shafer and A. Y. Rumfelt, IRE Trans. Microw. Theory Tech. MMT-\%, 447 (1959).

The treatment of mismatch errors is extended to cover variable attenuators cascade-connected in a system which is not free from reflections. The method of analysis is applicable to any number of cascaded attenuators, but only the analysis of two and three variable attenuators in cascade is presented. Graphs are given to aid in estimating the limits of mismatch error.

In an example, which is considered representative of rigid rectangular waveguide systems, the limits of error are: for two attenuators in cascade, $0.19 \mathrm{db}$ in a 3 -db measurement, and $0.17 \mathrm{db}$ in a $40-\mathrm{db}$ measurement; and for three attenuators in cascade, $0.25 \mathrm{db}$ in a $40-\mathrm{db}$ measurement, and $0.23 \mathrm{db}$ in a $75-\mathrm{db}$ measurement.

Use of Chebychev polynomials in thin film computations, K. D. Mielenz, J. Research NBS 63A, No. 3, 297 (1959).

From Herpin's expression for the $m$ th power of a multilayer matrix, very simple closed formulas are derived for the matrices and optical constants of any multilayer with a periodic structure.

According to Epstein's theorem, any symmetrical multilayer is equivalent to a fictitious monolayer. A simple expression for the equivalent index and thickness of this monolayer is deduced for the case of a periodic and symmetrical sequence of equally thick films.

As compared to any other method of numerical computation, the suggested formulation provides a considerable saving of time and work. In a numerical example, this saving amounts to about 80 percent.

Recent experimental evidence favouring the $\rho \boldsymbol{K}_{1}(\rho)$ correlation function for describing the turbulence of refractivity in the troposphere and stratosphere, K. A. Norton, J. Atmospheric and Terrest. Phys., 15, 206 (1959).

Experimental evidence of three different kinds is given which shows that the variations of the refractive index in space at a fixed time may be described with a useful degree of accuracy by the correlation function $\rho K_{1}(\rho)$ where $K_{1}$ is the modified Bessel function of the second kind and $\rho=r l_{0}$ is a normalized distance between two points in the atmosphere. The three kinds of evidence are (a) direct measurements of the variations of $n$ with time at a fixed location as made with a refractometer, (b) measurements of the variations with time of 